\title{
Review of: "Reconstructing cell interactions and state trajectories in pancreatic cancer stromal tumoroids"
}

\author{
Donald Gullberg ${ }^{1}$
}

1 University of Bergen

Potential competing interests: The author(s) declared that no potential competing interests exist.

The paper by Okuda et al outlines the use of stroma-rich PDAC tumoroid co-culture system in 3D composed of PDAC cells, endothelial cells and cancer-associated fibroblasts (CAFs) to study the effect of fibroblasts on cancer cells. The study involves a limited number of patient biopsies, but demonstrates the usefulness of this 3D system to dissect molecular mechanisms active in the parental tumors. Two interesting findings are notable:

-EGFR expression on CAFs,

- CEACAM6 expression on PDAC cancer cells.

EGFR is normally expressed on cancer cells where it has been shown to cooperate with integrins in signalling ${ }^{1}$. CEACAM6 which is a GPI anchored cell-cell adhesion molecule has previously been identified as a potential PDAC therapeutic target ${ }^{2}$.

The study is carefully conducted and encourages future use of tumoroids.

I have the following comments:

1. For the analysis of ECM genes, it will be important to follow up transcriptional data at protein level.

- ECM proteins are often composed of several protein subunits (including collagens which are homodimers or heterotrimers). For ECM protein levels to be increased it is required that all subunits are increased.

- It is also important to recognize that desmoplastic stroma (rich in fibrillar collagens) is different from basement membranes (containing laminin networks and the network forming collagen IV) associated with blood vessels.

2. It will also be important to more in detail analyze the receptors for ECM proteins, which are involved in creating downstream signalling and ECM structural changes, due to CAF-mediated ECM profile changes.

References cited:

1 Alfonzo-Mendez, M. A., Sochacki, K. A., Strub, M. P. \& Taraska, J. W. Dual clathrin and integrin signaling systems regulate growth factor receptor activation. Nat Commun 13, 905, doi:10.1038/s41467022-28373-x (2022).

2 Pandey, R. et al. Carcinoembryonic antigen cell adhesion molecule 6 (CEACAM6) in Pancreatic Ductal Adenocarcinoma (PDA): An integrative analysis of a novel therapeutic target. Sci Rep 9, 18347, doi:10.1038/s41598-019-54545-9 (2019). 
